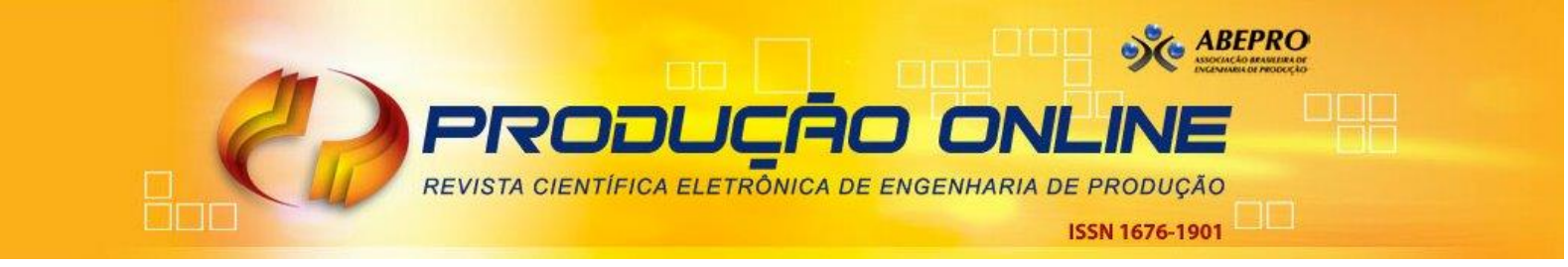

\title{
UTILIZAÇÃO DO AHP PARA PRIORIZAÇÃO DAS PRÁTICAS DE TQM NA INDÚSTRIA FARMACÊUTICA ${ }^{1}$
}

\section{USING THE AHP FOR PRIORITIZATION OF TQM PRACTICES IN PHARMACEUTICAL INDUSTRY}

\author{
Karla Silva Silveira*E-mail:karlasilveira.biotec@gmail.com \\ Suzana Eda Hikichi* E-mail: suzanaeda@hotmail.com \\ Eduardo Gomes Salgado*E-mail: eduardosalgado@unifal-mg.edu.br \\ * Universidade Federal de Alfenas, Campus Alfenas (UNIFAL-MG), Alfenas, MG
}

\begin{abstract}
Resumo: A Gestão pela Qualidade Total ou Total Quality Management (TQM) consiste em uma estratégia de gestão, que requer o envolvimento e comprometimento de toda organização, na melhoria contínua de produtos e processos, tendo como foco a satisfação do cliente. Este artigo destacou a importância de priorizar algumas práticas da Gestão da Qualidade Total na indústria farmacêutica, auxiliando na sua implantação. Foi utilizado o método de decisão multicriterial AHP (Analytic Hierarchy Process), o qual tem como função estruturar as práticas de TQM de forma hierarquizada. Assim foram avaliadas 17 práticas do TQM, divididas em três categorias (estratégicos, táticos e operacionais). Os resultados sugerem que a Gestão Estratégica foi o fator de maior importância, seguido pelos fatores táticos e operacionais. O presente trabalho atingiu seu objetivo de ajudar gestores do setor farmacêutico a se orientarem de forma eficaz para obter melhor desempenho das práticas do TQM e alcançar os objetivos desejados.
\end{abstract}

Palavras-chave: Gestão da Qualidade Total. Indústria farmacêutica. Hierarquia de processos. Práticas da qualidade. AHP.

Abstract: The Total Quality Management (TQM) consists in a management strategy, which requires the involvement and the commitment of the whole organization, in the continuous improvement of products and processes focusing on customer satisfaction. This paper highlighted the importance of prioritizing certain Total Quality Management practices at pharmaceutical industry, assisting in its implementation. The AHP (Analytic Hierarchy Process) multicriteria method was used, which has as its function structure the practices of TQM hierarchical form. So were evaluated 17 TQM practices, divided into three categories (strategic, tactical and operational). The results suggested the Strategic Management was the most important factor, followed by tactical and operational factors. The present work achieved its goal of helping managers in the pharmaceutical industry to be guided effectively to get better performance of TQM practices and achieve the desired objectives.

Keywords: Total Quality Management. Pharmaceutical Industry. Hierarchy Process. Quality practices. AHP.

\section{INTRODUÇÃO}

ambiente global na atualidade é marcado por mudanças em alta velocidade

1 O presente artigo é inédito, não foi financiado, é resultado de um Trabalho de Conclusão de Curso para a obtenção do Título de Bacharel e não apresenta conflitos de interesse.

Revista Produção Online, Florianópolis, SC, v. 16, n. 2, p. 524-549, abr.jun. 2016. 
e extrema competitividade. Empresas que almejam atingir benefícios relacionados à produtividade e aumento da participação no mercado necessitam de constante modernização e expansão de sua capacidade de aprendizagem e inovação. Nesse âmbito, a implantação de Sistemas de Gestão da Qualidade (SGQ), com suas ferramentas da qualidade, constitui uma prática indispensável no processo de crescimento de organizações, garantindo a qualidade, a atratividade e a confiabilidade de seus produtos no mercado consumidor.

A preocupação com a gestão da qualidade propiciou o surgimento de ferramentas (técnicas e métodos) que possibilitam gerir, controlar e aperfeiçoar os processos executados nas mais diferentes tipos de organizações, além de prever a eliminação ou simplificação de processos que não adicionam valor ao produto final.

Modelos difundidos e aplicados, como a certificação de sistemas de qualidade pelas normas ISO 9000 e Seis Sigma, e a estratégia de gestão Total Quality Management (TQM) ou Gestão da Qualidade Total, possibilitam às empresas uma melhoria contínua dos processos organizacionais, criando vantagem competitiva e adaptação às diferentes exigências do mercado (FASSOULA, 2006). O avanço da filosofia de gestão TQM ocorreu devido a essas tentativas de melhoria da qualidade nas organizações (EHIGIE; MCANDREW, 2005).

$\mathrm{Na}$ literatura sobre o tema, muitas práticas associadas ao TQM podem ser identificadas em diferentes setores. Esse grande número de fatores e elementos relacionados, no entanto, vem tornando implantação do TQM cada vez mais complicada, e exigindo cada vez mais a priorização dessas práticas, iniciativa que muitas vezes é preterida dentro das empresas (CHIN et al, 2002; TALIB; RAHMAN; QURESHI, 2011; SARATHY, 2013).

Dessa forma, torna-se crítico determinar, entre o universo de práticas, aquelas que contribuem de forma mais significativa para o sucesso da implantação do TQM. A priorização dessas práticas garante maior adequação entre a alocação de recursos e os resultados alcançados. Além disso, de acordo com Bayazita e Karpak (2007), é considerável o número de organizações que falham na tentativa de implantação dessas práticas, enquanto muitas outras obtêm sucesso, justificando-se a realização de pesquisas sobre esse tema.

O setor da saúde corresponde a um segmento de grande importância na qualidade de vida da população mundial, responsável pela pesquisa, fabricação e 
comercialização de produtos utilizados no diagnóstico e tratamento de doenças. Assim, espera-se que a indústria farmacêutica, responsável pelo desenvolvimento de medicamentos, garanta a eficácia e segurança de seus produtos (MAZUMDER; BHATTACHARYA; YADAV, 2011; BAUCHNER; FONTANAROSA, 2013).

A gestão da qualidade na indústria farmacêutica baseia-se na prática de ferramentas da qualidade durante todas as fases de produção. Ações sistemáticas e precisas devem ser tomadas para assegurar que determinado produto (ou processo) satisfaça as exigências quanto à sua qualidade (SINGH; DHALLA, 2010). Dessa forma, a Gestão da Qualidade Total (TQM) surge como uma grande alternativa para a busca de qualidade com melhoria contínua, possibilitando um retorno de desempenho e redução de custos para uma melhoria de produtividade e competitividade (MAZUMDER; BHATTACHARYA; YADAV, 2011).

Embora sejam desenvolvidos muitos estudos a respeito da adoção das práticas do TQM no setor farmacêutico, pouca atenção tem sido dada, assim como na indústria em geral, ao ato de priorizar essas práticas. A grande importância social e econômica desse setor, entretanto, motiva a realização de estudos para determinar a prioridade da implantação das práticas do TQM nessas indústrias. Para isso, serão avaliadas as práticas de Gestão da Qualidade Total estudadas por Talib, Rahman e Qureshi (2011), Khanna, Sharma e Laroiya (2011), Lewis, Pun e Lalla (2005), Kumar, Garg e Garg, (2011) e Souza Junior et al. (2014).

Para a realização da priorização das práticas no setor industrial farmacêutico, diferentes métodos de tomadas de decisão podem ser utilizados. Entre eles, destaca-se o Analytic Hierarchy Process (AHP) (SAATY, 1980), uma ferramenta para tomada de decisões e avaliação de critérios utilizando julgamentos de especialistas (CASTROGIOVANNI et al., 2015). Ela vem sendo amplamente empregada por ser de fácil uso, lógica e transparente, adequada para melhorar a compreensão e avaliação da pesquisa, e por auxiliar na tomada de decisões quando existem diversos fatores a serem considerados simultaneamente.

Por conseguinte, o presente trabalho possui como objetivo principal a aplicação do método AHP na realização da priorização das práticas da Gestão da Qualidade Total (TQM) em uma indústria farmacêutica. De forma complementar, o estudo também buscou auxiliar a empresa selecionada e outras organizações do 
seu setor a controlarem suas atividades diárias, buscando melhorar seu desempenho no gerenciamento da qualidade total.

\section{REVISÃO DE LITERATURA}

\subsection{A qualidade na indústria farmacêutica}

O setor farmacêutico representa um segmento crucial na área da saúde, contribuindo em muitos avanços que permitiram um expressivo aumento da qualidade e expectativa de vida dos seres humanos nas últimas décadas. Inovações como a descobertas de drogas como aspirina, antibióticos e estatinas, a fabricação e comercialização de inúmeros produtos farmacêuticos e biológicos, e o desenvolvimento de novas tecnologias utilizadas no diagnóstico e tratamentos de doenças mostram a grande importância desse setor industrial na atualidade (KOLA; LANDIS; 2004; MAZUMDER; BHATTACHARYA; YADAV, 2011).

Em paralelo, os desafios enfrentados pelas companhias farmacêuticas nunca foram tão grandes. Os custos farmacêuticos têm crescido de forma contínua, representando uma parcela significativa do PIB nacional dos países (MEHRALIAN et al., 2015). As indústrias do setor têm confrontado severos problemas relacionados a qualidade de seus produtos, representado pelo alto número de recalls de medicamentos, relacionados principalmente à baixa qualidade da matéria-prima, problemas no empacotamento e contaminação na produção, ou seja, às práticas durante o processo de manufatura (FRIEDLI; GOETZFRIED; BASU, 2010). A precária qualidade desses medicamentos, além de representar sérios riscos à saúde tem provocado prejuízos para governos e empresas, e aumento no custo para o consumidor final.

Ao mesmo tempo, esse setor também vem apresentando outros sérios problemas políticos e fiscais, como a redução no orçamento com a saúde pelos governos dos países e a necessidade de mudanças estruturais, acompanhada pela elevação nos custos do desenvolvimento de novas drogas (KOLA; LANDIS; 2004; SCHNEIDER et al., 2015). Além disso, diversos fatores como pressões de competidores externos, a fabricação de medicamentos genéricos e a maior 
exigência do consumidor também vêm impulsionando as indústrias a buscarem alternativas para reduzir seus custos de produção (McADAM; BARRON, 2002).

Diante desse cenário, o tópico qualidade vem se tornando fundamental dentro da indústria farmacêutica, e a aplicação de sistemas de gestão visando a manutenção da qualidade com melhoria da eficácia e eficiência operacional tem se popularizando no setor (FRIEDLI; GOETZFRIED; BASU, 2010). A prática da Gestão da Qualidade Total (TQM) constitui um esforço que tem auxiliado diversas organizações a manter a alta qualidade dos seus produtos e serviços, satisfazendo os clientes e melhorando sua competitividade dentro do mercado (MEHRALIAN et al., 2015).

\subsection{Gestão da Qualidade Total (TQM)}

A prática da Gestão da Qualidade Total foi desenvolvida inicialmente por autores norte-americanos como Deming, Juran e Feigenbaum, em meados da década de 1940, encontrando no Japão o ambiente perfeito para o seu desenvolvimento, nos anos posteriores. Desta forma, em 1980 a indústria japonesa passou a se destacar no mundo todo, com produtos de excelente qualidade e preços relativamente baixos no mercado, despertando grande interesse relacionado ao TQM nas empresas norte-americanas e europeias (CARVALHO; PALADINI, 2005).

O TQM consiste em uma estratégia de gestão que busca, de forma contínua, a melhoria da qualidade de produtos e processos, focando na satisfação do cliente. A sua implantação permite à organização aumentar a produtividade interna, assim como a sua competividade dentro do mercado no qual ela está inserida (CHEN; CHEN, 2009; KHANNA et al., 2011). Assim, o TQM é variável e adaptável, podendo ser definido como um método gerencial para melhorar tanto capacidade competitiva de uma organização como para maximizar a participação desta no mercado na qual ela está inserida (KUMAR, GARG; GARG, 2011, CHEN; CHEN, 2009).

Essa estratégia é baseada na participação e dedicação de todos os setores da empresa, sendo necessária uma grande contribuição da alta gerência, comprometimento de todos os empregados, treinamento e trabalho em equipe, buscando resultados com qualidade elevada, para que a organização seja bem sucedida em todas as iniciativas isoladas ou em conjunto (KUMAR et al., 2011). 
Entre os benefícios que o TQM proporciona estão (LEWIS et al. 2005; KUMAR et al., 2011):

- melhoria contínua aos produtos e processos;

- aumento da rentabilidade;

- estímulo ao crescimento da produtividade;

- minimiza exponencialmente os custos internos;

- satisfação dos clientes internos e externos;

- mão de obra treinada;

- capacitação de empregados;

- redução do desperdício;

- poluição reduzida.

O ciclo PDCA (Plan, Do, Check, Act) pode ser considerado um dos métodos de gestão que a empresa pode utilizar para alcançar o TQM. Ele permite o gerenciamento de todos os seus processos, garantindo que as metas determinadas pela organização sejam atingidas (CAMPOS, 2004).

Explorando a literatura sobre o TQM, uma ampla gama de estudos pode ser encontrada. Fotopoulos e Psomas (2010) avaliaram a relação entre os fatores do TQM e a performance organizacional. Hung et al. (2011) avaliaram o efeito da relação entre $\mathrm{O} T Q M$ e a performance organizacional sobre a performance de inovações das indústrias. Ooi et al. (2011) realizaram um estudo empírico para investigar a associação entre as práticas do TQM com a qualidade dos serviços e a satisfação dos consumidores em organizações da Malásia. Mosadeghrad (2013) analisou quais os fatores dificultavam a implantação do TQM e a alta taxa de falhas nessa implantação dentro do setor de cuidados com a saúde. Miranda Silva et al. (2014) estudaram por sua vez os efeitos da implantação do TQM na estratégia de inovação do produto. Lau, Tang e Li (2015) estudaram o nível de aplicação do TQM por empreiteiras da área da construção em Hong Kong.

Na bibliografia da Gestão da Qualidade Total, muito tem sido descrito sobre o que constituem os ingredientes básicos ou os pilares filosóficos do TQM. O número e a prioridade desses elementos variam de um autor para outro e sua importância pode variar também de uma organização para outra. Além disso, segundo Harrington, Voeh e Wiggin (2012) a maior parte da literatura que aborda os elementos do TQM estaria desatualizada. 


\subsection{Método Analytic Hierarchy Process (AHP)}

De acordo com Shimizu (2006) o processo de tomada de decisão envolve, quase sempre, a escolha da melhor decisão levando em conta múltiplos critérios, fatores e objetivos. Os métodos de aperfeiçoamento da programação matemática podem ser inadequados para tratar o caso do problema com múltiplos critérios e variáveis qualitativas.

O método AHP, desenvolvido por Thomas L. Saaty na década de 1970 tem como objetivo auxiliar na tomada de decisão, a fim de resolver problemas complexos e não estruturados. Ele constitui uma ferramenta multicriterial de tomada de decisão, que através de um problema, o objetivo geral, e do desenvolvimento de critérios, subcritérios e de análises, permite a tomada de decisão (SAATY, 1988).

Essa estratégia multicriterial permite que o usuário atribua pesos relativos para variados critérios, de forma intuitiva, realizando também uma comparação par a par entre os mesmos. Permite também que, mesmo quando duas variáveis são incomparáveis, com os conhecimentos e a experiência das pessoas, seja possível reconhecer qual dos critérios é o mais importante. (SAATY, 1991). Esse método representa uma melhoria para a tomada de decisões por gestores, que se baseiam muitas vezes em suas experiências ou sentimentos (SALGADO et al., 2015).

O AHP foi escolhido como o método de auxílio à decisão a ser utilizado na pesquisa em questão para seleção das etapas do modelo proposto. De acordo com Braglia et al. (2006), ele representa uma das abordagens mais seguras para a decisão de múltiplos critérios.

Segundo Sipahi e Timor (2010), no período de 2005 a 2009, mais de 600 artigos foram publicados relacionados com o AHP ou com o Analytic Network Process (ANP), sendo relatado que 0 uso do AHP está crescendo exponencialmente. O ANP constitui uma extensão do AHP, que permite a análise da dependência entre critérios ou alternativas.

A ferramenta AHP vem sendo amplamente aplicada em diversas áreas do conhecimento. Entre as várias utilizações do AHP, Costa et al. (2008) empregaram a ferramenta para avaliar as características das interfaces telefônicas utilizadas em uma determinada empresa de telefonia no serviço de atendimento ao cliente. Através de uma análise criteriosa foi possível verificar qual solução tecnológica 
oferece vantagem para a empresa e satisfação ao cliente. Um processo AHP foi usado por Gupta et al. (2015) para avaliar diferentes práticas sustentáveis de produção em indústrias de painéis elétricos na Índia. Singh e Nachtnebel (2016) aplicaram o AHP para classificar hidrelétricas considerando os critérios social, econômico e ambiental.

Leite e Freitas (2012) afirmam que o AHP seria o processo mais apropriado, em comparação com dois principais métodos de apoio à tomada de decisão, Elimination and Choice Expressing Reality (ELECTRE) e Preference Ranking Organisation Method for Enrichment Evalutations (PROMETHEE), quando os critérios são passíveis de comparação e possuem uma estrutura hierárquica. $O$ Quadro 1 apresenta alguns autores que vêm aplicando os métodos citados de tomada de decisão, assim como aqueles que têm utilizado outros dois métodos amplamente divulgados, MAUT e TOPSIS.

\begin{tabular}{|c|c|c|}
\hline $\begin{array}{l}\text { Métodos de } \\
\text { Tomada de } \\
\text { Decisão }\end{array}$ & Definição & Alguns autores que utilizam o método \\
\hline ELECTRE & $\begin{array}{l}\text { Elimination and Choice } \\
\text { Expressing Reality }\end{array}$ & $\begin{array}{l}\text { Tam, Tong e Lau (2003); López, Sánchez e } \\
\text { Contreras (2008); Mota e Almeida (2007); } \\
\text { Rangel, Gomes e Moreira (2009). }\end{array}$ \\
\hline PROMETHEE & $\begin{array}{l}\text { Preference Ranking } \\
\text { Method for Enrichment } \\
\text { Evaluation }\end{array}$ & $\begin{array}{l}\text { Anandi e Kodali (2008); Cavalcante e Almeida } \\
\text { (2007); Cavalcante e Almeida (2005); Ballis e } \\
\text { Mavrotas (2007); Koutroumanidis, } \\
\text { Papathanasiou e Manos (2002). }\end{array}$ \\
\hline MAUT & $\begin{array}{l}\text { Multiple attribute utility } \\
\text { theory }\end{array}$ & Chang (2009); Collins et al. (2006); \\
\hline TOPSIS & $\begin{array}{lrr}\text { Technique for } & \text { Order } \\
\text { Preference } & & \text { by } \\
\text { Similarity to } & \text { Ideal } \\
\text { Solution } & & \end{array}$ & $\begin{array}{l}\text { Kahraman, et al. (2007); Bottani e Rizzi (2006); } \\
\text { Nejati e Nejati (2009); Jamali e Nejati (2009); } \\
\text { Polychroniou e Giannikos (2009); }\end{array}$ \\
\hline
\end{tabular}

Fonte: Autores 
Os exemplos citados mostram que, com uma simples comparação par a par dos dados, é possível tomar uma decisão focalizando cada parte e todo o complexo problema através de prioridades.

O método AHP fundamenta-se em três etapas: construção de hierarquias através da estruturação do problema, estabelecimento de pesos para critérios e preferência para as alternativas e por fim, análise dos resultados. (TRAMARICO; SALOMON; MARINS, 2012).

$\mathrm{Na}$ construção de hierarquias o problema é estruturado em níveis hierárquicos, permitindo uma melhor compreensão e também a avaliação do mesmo. Os critérios e as alternativas são estruturados de forma hierárquica conforme apresenta a Figura 1, sendo que o primeiro nível da hierarquia corresponde ao objetivo geral, o segundo nível aos critérios e o terceiro, às alternativas (subcritérios) (SAATY, 1980). Segundo Bornia (2001), a construção de hierarquias possibilita uma melhor visualização global do problema e da relação de complexidade envolvida para resolvê-lo.

Figura 1- Estrutura hierárquica genérica

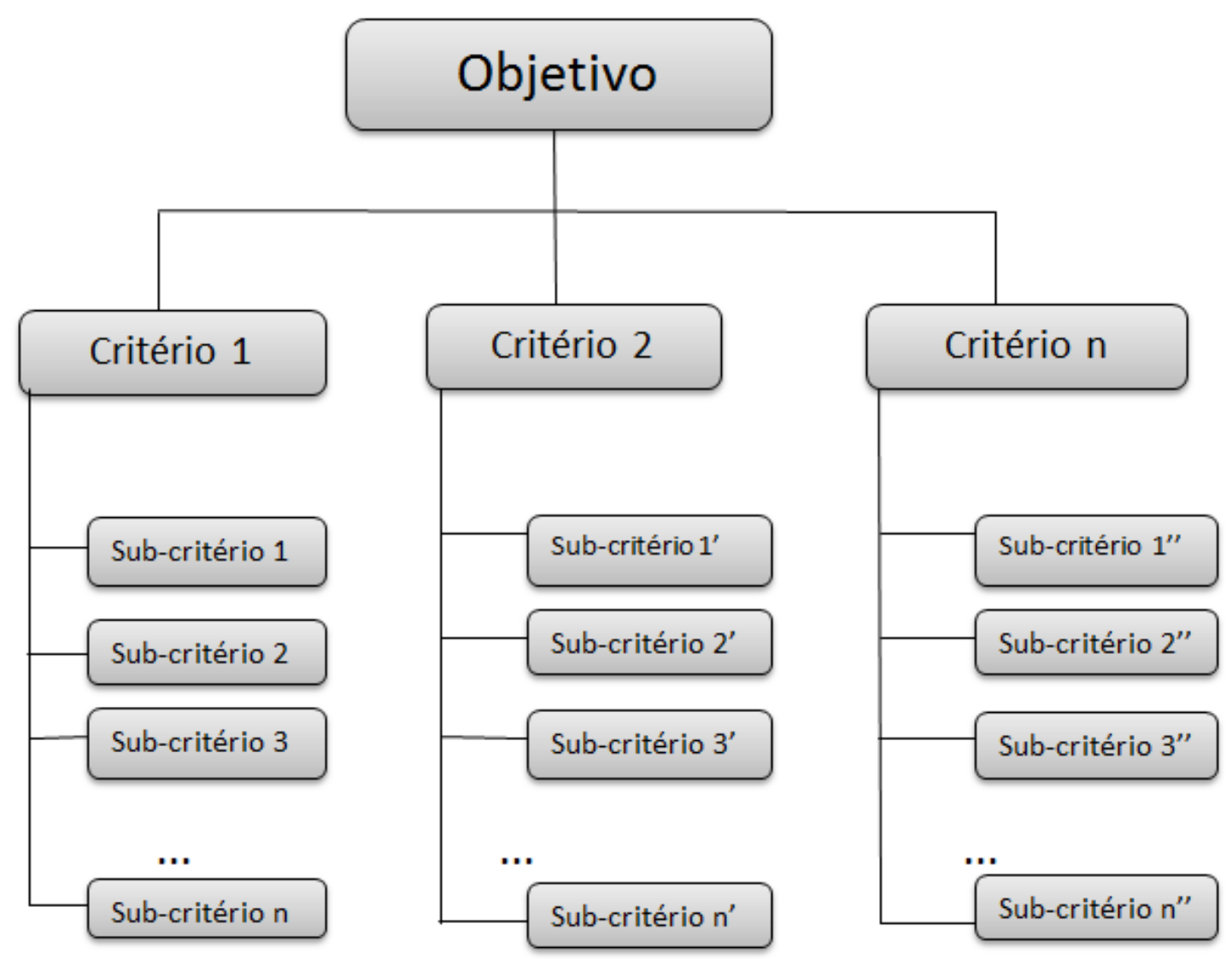

Fonte: Salgado et al. (2012)

Revista Produção Online, Florianópolis, SC, v. 16, n. 2, p. 524-549, abr.jun. 2016. 
A Figura 1 apresenta uma estrutura hierárquica genérica. Segundo Bornia (2001), essa construção de hierarquias possibilita uma melhor visualização global do problema e da relação de complexidade envolvida para resolvê-lo.

$\mathrm{Na}$ etapa de definição de prioridades é realizada uma comparação par a par dos dados, criando assim uma matriz de comparações entre os critérios, avaliando a importância de uma característica sobre a outra (TRAMARICO; SALOMON; MARINS, 2012).

Para essas comparações Saaty (1980), propôs a utilização de uma escala linear de 1 a 9, denominada de Escala Fundamental de Números Absolutos (SAATY, 2005). A Tabela 1 apresenta essa escala de valores que deverá ser utilizada para a comparação entre os pares.

Tabela 1 - Escala de comparação de critérios.

\begin{tabular}{cl}
\hline Intensidade da importância & \multicolumn{1}{c}{ Explicação } \\
\hline 1 & Ambos os elementos são de igual importância \\
3 & Moderada importância de um elemento sobre o outro. \\
5 & Forte importância de um elemento sobre o outro \\
7 & Importância muito forte de um elemento sobre o outro \\
9 & Extrema importância de um elemento sobre o outro. \\
$2,4,6,8$ & Valores intermediários entre as opiniões adjacentes \\
\hline
\end{tabular}

Fonte: Saaty (2009)

A reciprocidade dessas comparações, ou seja, $a_{i j}=1 / a_{j i}$ deverá ser considerada após a comparação através da Escala Fundamenta de Saaty. Como as matrizes de comparações são preenchidas por completo, podem ocorrer comparações redundantes, conhecido como Relação de Transitividade (GOMES et al., 2009).

A matriz de comparações que satisfaz as relações de transitividade tem $100 \%$ de consistência. O autovalor dessa matriz será $\lambda \max =n$, sendo $n$ a ordem da matriz. Já o índice de consistência é $\mu=(\lambda \max -n) /(n-1)$ e refere-se a confiabilidade da informação fornecida para o preenchimento da matriz de comparações (SAATY, 1997).

Para a realização desse método pode ser considerado apenas um único julgador ou também um grupo de indivíduos, sendo fornecidas assim $\mathrm{N}$ matrizes de 
julgamento para cada um, contudo somente uma matriz será utilizada, obtida pela média aritmética (BASAK; SAATY, 1993).

Esse processo pode ser aplicado a diferentes setores. Hsu Tzeng e Shyu (2003) selecionaram os principais projetos a serem desenvolvidos pelo governo, diante de diferentes grupos de interesse, aplicando essa análise. Salmeron e Herrero (2005) realizaram a priorização dos fatores críticos de sucesso no desenvolvimento e implantação de sistemas de informação.

Entre outros exemplos ainda estão Kang e Lee (2007), que apresentaram outra utilização do método, desenvolvendo um estudo para o processo de fabricação de semicondutores, possibilitando a resolução de problemas que apresentam múltiplos critérios e permitindo assim a tomada de decisões. Nikou e Mezei (2013) utilizaram a estratégia para avaliar o processo de adoção de serviços móveis como Short Messaging (SMS) e e-mail móvel, através da priorização dos serviços móveis e dos fatores que impulsionam a adoção pelos consumidores.

$\mathrm{Na}$ mesma linha de raciocínio, Salgado et al. (2012) utilizaram o AHP na identificação de atividades críticas do desenvolvimento de novos produtos. Sarathy (2013) aplicou a ferramenta na avaliação de praticas de gestão da qualidade na indústria de imóveis e Souza Jr. et al. (2014), realizaram a priorização de práticas do TQM em uma indústria farmacêutica. Mota e Almeida (2007) apresentaram em seu trabalho um modelo multicritério para tratar o problema de priorização de atividades em projetos por meio de análise dos fatores considerados críticos, numa visão integrada dos empreendimentos.

A literatura do TQM por sua vez, em geral apresenta poucos estudos relacionados ao uso de uma abordagem multicritério para o tratamento de priorização das práticas da Gestão da Qualidade Total.

\section{METODOLOGIA}

\subsection{Estudo de caso}

Para Martins (2010), os métodos de pesquisa mais apropriados para conduzir uma pesquisa qualitativa são: estudo de caso e pesquisa-ação. Dessa forma na presente pesquisa será utilizado o estudo de caso. 
Segundo Voss, Tsikriktsis e Frohlich (2002), existem muitos desafios na condução de um estudo de caso: ele consome muito tempo, necessita de entrevistadores com habilidade e conhecimento, é necessário muito cuidado na generalização das conclusões a partir de um limitado conjunto de casos e na garantia do rigor da pesquisa. Apesar disso, os resultados de um estudo de caso podem ter um forte impacto e levar a novas e criativas percepções e a se desenvolver novas teorias. Além disso, Yin (2005) afirma que a investigação utilizando o estudo de caso enfrenta uma situação única em que haverá muito mais variáveis de interesse do que pontos de dados.

Dessa forma, Eisenhardt (1989) definiu as fases necessárias para a condução do estudo de caso, que são: definição da questão da pesquisa, seleção dos casos, criação do instrumento de pesquisa e protocolo, condução dar pesquisa de campo, análise dos dados, formulação da hipótese, comparação com a literatura e conclusão da pesquisa. Seguindo a mesma linha do autor anterior, Miguel (2007) propôs as seguintes macro etapas para a condução do estudo de caso: definição de uma estrutura conceitual teórica, planejamento do(s) caso(s), condução do teste piloto, coleta dos dados, análise dos dados e redação do relatório.

A decisão sobre a realização de um caso único ou casos múltiplos deve acontecer antes da coleta de dados. A utilização de múltiplos casos possui vantagens e desvantagens em comparação a um único caso. Dessa forma, foi utilizado o AHP como ferramenta para a entrevista realizada.

\subsection{Aplicação do método AHP}

A indústria farmacêutica em estudo está localizada na região Sul de Minas Gerais, presente há 15 anos no mercado, com um quadro de em média 800 funcionários. Representa uma das maiores empresas na produção de medicamentos de uso hospitalar no Brasil, atuando também nos segmentos oftalmológico, sistema nervoso central, genéricos, entre outros.

No presente estudo, foram realizados os 4 passos de acordo com a metodologia (AHP) estabelecida por Saaty (1988):

\section{- Passo 1: Definir o objetivo do estudo:}


O objetivo do estudo foi determinar as prioridades da implantação das práticas de TQM em uma determinada indústria farmacêutica, para melhorar o seu desempenho na sua gestão da qualidade, buscando alcançar o máximo de benefícios com essa prática.

\section{- Passo 2: Decompor o objetivo em critérios e subcritérios:}

O objetivo foi decomposto em três fatores (critérios) e 17 práticas de TQM (subcritérios), identificados a partir dos estudos de Talib, Rahman e Qureshi (2011) e Souza Jr et al. (2014), formando assim uma estrutura hierárquica.

Os fatores (critérios) identificados foram os seguintes:

1. Fatores estratégicos.

2. Fatores táticos.

3. Fatores operacionais.

As práticas de TQM (subcritérios) determinadas foram as seguintes:

a) Fatores Estratégicos: compromisso da direção, cultura da qualidade, melhoria contínua e inovação, sistemas de qualidade, Benchmarking e planejamento estratégico.

b) Fatores Táticos: incentivo aos trabalhadores, envolvimento dos trabalhadores, treinamento e educação, trabalho em equipe, informação e análise, gestão de fornecedores e comunicação.

c) Fatores Operacionais: design do produto, gestão de processo, foco no cliente e gestão de recursos humanos.

- Passo 3: Construir uma estrutura de hierarquia para análise:

A estrutura hierárquica para o estudo, construída a partir dos critérios e subcritérios identificados é apresentada na Figura 2: 
Figura 2 - Estrutura hierárquica

\section{Priorização das práticas de TQM}

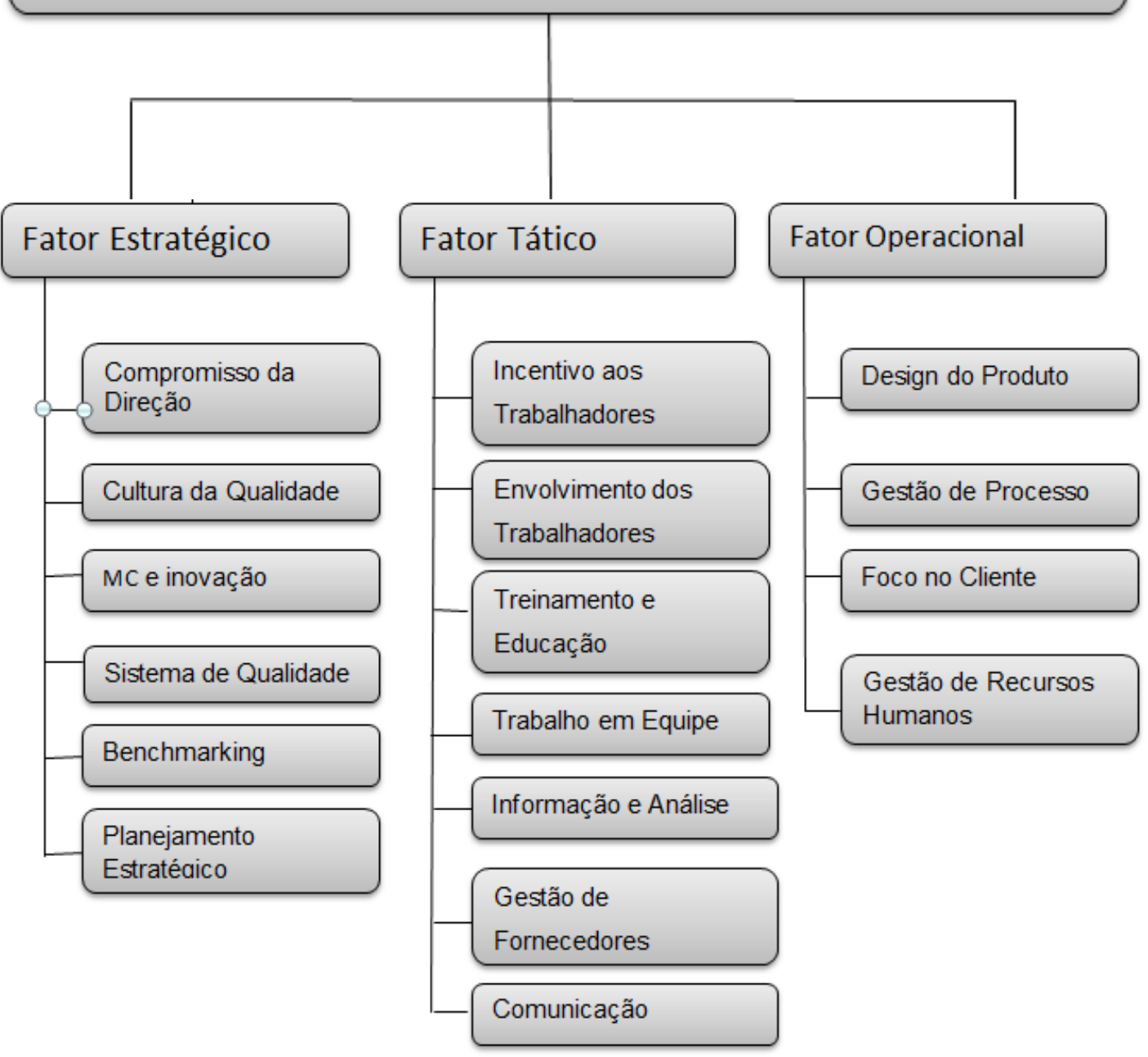

Fonte: Talib, Rahman e Qureshi (2011)

\section{- Passo 4: Coleta de informações:}

Essa etapa corresponde à coleta de informações, obtida através de julgamentos dos avaliadores de uma determinada indústria farmacêutica. Neste estudo, foi selecionado e entrevistado um grupo de 12 pessoas, que avaliaram todos os critérios e subcritérios. O grupo de avaliadores é composto por líderes, analistas, coordenadores e gerentes, com experiência na área de gestão da qualidade.

Nos Quadros 2, 3 e 4 a seguir encontram-se as características individuais de cada avaliador. 
Quadro 2- Características dos especialistas avaliados

\begin{tabular}{|l|l|l|l|l|}
\hline Característica & \multicolumn{1}{|c|}{$\begin{array}{c}\text { Entrevistado } \\
\text { A }\end{array}$} & Entrevistado B & Entrevistado C & $\begin{array}{l}\text { Entrevistado } \\
\text { D }\end{array}$ \\
\hline $\begin{array}{l}\text { Tempo na } \\
\text { função }\end{array}$ & 12 anos & 4 anos & 10 anos & 7 anos \\
\hline $\begin{array}{l}\text { Formação } \\
\text { acadêmica }\end{array}$ & $\begin{array}{l}\text { Superior em } \\
\text { Farmácia. }\end{array}$ & $\begin{array}{l}\text { Superior em Eng. } \\
\text { de Produção. }\end{array}$ & $\begin{array}{l}\text { Superior em } \\
\text { Farmácia. }\end{array}$ & $\begin{array}{l}\text { Superior em } \\
\text { Farmácia }\end{array}$ \\
\hline Função & Gerente. & Supervisor. & Coordenador. & Coordenador \\
\hline $\begin{array}{l}\text { Área de } \\
\text { trabalho }\end{array}$ & $\begin{array}{l}\text { Controle de } \\
\text { Qualidade. }\end{array}$ & $\begin{array}{l}\text { Garantia da } \\
\text { Qualidade. }\end{array}$ & $\begin{array}{l}\text { Controle de } \\
\text { Qualidade. }\end{array}$ & $\begin{array}{l}\text { Controle de } \\
\text { Qualidade. }\end{array}$ \\
\hline
\end{tabular}

Fonte: Autores

Quadro 3- Características dos especialistas avaliados

\begin{tabular}{|l|l|l|l|l|}
\hline Característica & Entrevistado E & Entrevistado F & Entrevistado G & \multicolumn{1}{|l|}{$\begin{array}{l}\text { Entrevistado } \\
\text { H }\end{array}$} \\
\hline $\begin{array}{l}\text { Tempo na } \\
\text { função }\end{array}$ & 3 anos & 2 anos & 5 anos & 3 anos \\
\hline $\begin{array}{l}\text { Formação } \\
\text { acadêmica }\end{array}$ & $\begin{array}{l}\text { Superior em } \\
\text { Farmácia. }\end{array}$ & $\begin{array}{l}\text { Superior em } \\
\text { Farmácia. }\end{array}$ & $\begin{array}{l}\text { Superior em } \\
\text { Farmácia. }\end{array}$ & $\begin{array}{l}\text { Superior em } \\
\text { Farmácia. }\end{array}$ \\
\hline Função & Supervisor. & Analista Sr. & Analista. & Analista. \\
\hline $\begin{array}{l}\text { Área de } \\
\text { trabalho }\end{array}$ & $\begin{array}{l}\text { Controle de } \\
\text { Qualidade. }\end{array}$ & Reclamações. & $\begin{array}{l}\text { Controle de } \\
\text { Qualidade. }\end{array}$ & $\begin{array}{l}\text { Controle de } \\
\text { Qualidade. }\end{array}$ \\
\hline
\end{tabular}

Fonte: Autores

Quadro 4- Características dos especialistas avaliados

\begin{tabular}{|l|l|l|l|l|}
\hline Característica & Entrevistado I & Entrevistado J & $\begin{array}{l}\text { Entrevistado } \\
\text { K }\end{array}$ & Entrevistado L \\
\hline $\begin{array}{l}\text { Tempo na } \\
\text { função }\end{array}$ & 3 anos & 4 anos & 2 anos & 2 anos \\
\hline $\begin{array}{l}\text { Formação } \\
\text { acadêmica }\end{array}$ & $\begin{array}{l}\text { Superior em } \\
\text { Farmácia. }\end{array}$ & $\begin{array}{l}\text { Superior em } \\
\text { Administração. }\end{array}$ & $\begin{array}{l}\text { Gestão de de } \\
\text { produção. }\end{array}$ & $\begin{array}{l}\text { Tecnólogo em } \\
\text { Produção }\end{array}$ \\
\hline Função & Analista. & Analista. & Líder de linha. & Líder de linha. \\
\hline $\begin{array}{l}\text { Área de } \\
\text { trabalho }\end{array}$ & Lean/ Produção & Lean/ Produção & $\begin{array}{l}\text { Lean/ } \\
\text { Produção }\end{array}$ & Lean/ Produção \\
\hline
\end{tabular}

Fonte: Autores

Inicialmente foi realizado o julgamento de importância para os três critérios (primeiro nível), através da comparação em pares (TABELA 2): 
Tabela 2 - Julgamentos dos Critérios - Especialista: Supervisor no Controle de Qualidade

\begin{tabular}{ccccc}
\hline Critérios do TQM & FE & FT & FO & Prioridade \\
\hline Fatores Estratégicos (FE) & 1 & 3 & 3 & $59,78 \%$ \\
Fatores Táticos (FT) & $1 / 3$ & 1 & 2 & $22,83 \%$ \\
Fatores Operacionais (FO) & $1 / 3$ & $1 / 2$ & 1 & $17,39 \%$ \\
\hline
\end{tabular}

Fonte: Autores

Posteriormente foi realizado o julgamento da importância para os subcritérios, através da comparação em pares. Foram obtidos os resultados dos julgamentos relacionados aos critérios estratégicos, táticos e operacionais, apresentados respectivamente nas Tabelas 3, 4 e 5, juntamente com suas prioridades.

Tabela 3 - Julgamentos dos Fatores estratégicos - Especialista: Supervisor no Controle de Qualidade

\begin{tabular}{cccccccc}
\hline Fatores Estratégicos & CD & CQ & MCI & SQ & BM & PE & Prioridade \\
\hline Compromisso da Direção (CD) & 1 & 2 & 2 & $1 / 2$ & 3 & $1 / 3$ & $21,69 \%$ \\
Cultura da Qualidade (CQ) & $1 / 2$ & 1 & $1 / 3$ & $1 / 3$ & 5 & $1 / 3$ & $5,95 \%$ \\
Melhoria Continua e Inovação (MCI) & $1 / 2$ & 3 & 1 & $1 / 3$ & 4 & $1 / 5$ & $7,70 \%$ \\
Sistema da Qualidade (SQ) & 2 & 3 & 3 & 1 & 6 & $1 / 3$ & $24,45 \%$ \\
Benchmarking (BM) & $1 / 3$ & $1 / 5$ & $1 / 4$ & $1 / 6$ & 1 & $1 / 6$ & $3,25 \%$ \\
Planejamento Estratégico (PE) & 3 & 3 & 5 & 3 & 6 & 1 & $36,96 \%$
\end{tabular}

Fonte: Autores

Tabela 4 - Julgamentos dos Fatores táticos - Especialista: Gerente no Controle de Qualidade

\begin{tabular}{ccccccccc}
\hline Fatores Táticos & IT & EE & TE & TEQ & IA & GF & CO & Prioridade \\
\hline Incentivo aos Trabalhadores & 1 & 1 & 1 & 3 & 2 & $1 / 3$ & 2 & $13,77 \%$ \\
Envolvimento dos Empregados & 1 & 1 & 1 & 2 & 3 & $1 / 3$ & $1 / 2$ & $11,90 \%$ \\
(EE) & 1 & 1 & 1 & 3 & $1 / 3$ & $1 / 2$ & 1 & 12,87 \\
Treinamento e Educação (TE) & 1 & $1 / 3$ & $1 / 3$ & 1 & $1 / 3$ & $1 / 5$ & $1 / 5$ & $4,86 \%$ \\
Trabalho em Equipe (TEQ) & $1 / 3$ & $1 / 2$ & $1 / 3$ & $1 / 2$ & $10,03 \%$ \\
Informações e Análise (IA) & $1 / 2$ & $1 / 3$ & 3 & 3 & 1 & $1 / 5$ & $1 / 2$ \\
Gestão de Fornecedores (GF) & 3 & 3 & 2 & 5 & 5 & 1 & 3 & $33,54 \%$ \\
Comunicação (CO) & $1 / 2$ & 2 & 1 & 5 & 2 & $1 / 3$ & 1 & $13,03 \%$ \\
\hline
\end{tabular}

Fonte: Autores 
Tabela 5 - Julgamentos dos Fatores operacionais - Especialista: Gerente no Controle de Qualidade

\begin{tabular}{cccccc}
\hline Fatores Operacionais & DP & GP & FC & RH & Prioridade \\
\hline Design do Produto (DP) & 1 & $1 / 5$ & $1 / 5$ & $1 / 5$ & $7,31 \%$ \\
Gestão de Processo (GP) & 5 & 1 & $1 / 2$ & 3 & $28,72 \%$ \\
Foco no Cliente (FC) & 5 & 2 & 1 & 3 & $48,87 \%$ \\
Gestão de Recursos Humanos (RH) & 5 & $1 / 3$ & $1 / 3$ & 1 & $15,10 \%$ \\
\hline
\end{tabular}

Fonte: Autores

\section{RESULTADOS E DISCUSSÕES}

Com as informações obtidas nas entrevistas com os profissionais, foi realizada a priorização das práticas de TQM, como apresentado na Tabela 6, determinando a média local dos valores e sua média global.

Tabela 6 - Resultados Gerais da priorização das práticas de TQM

\begin{tabular}{|c|c|c|c|c|c|}
\hline Nível & $\begin{array}{c}\text { Critérios das práticas de } \\
\text { TQM }\end{array}$ & $\begin{array}{l}\text { Peso } \\
\text { Local }\end{array}$ & Ranking & $\begin{array}{l}\text { Peso } \\
\text { Geral }\end{array}$ & Ranking \\
\hline Critério & Fatores estratégicos & $58,29 \%$ & & $58,29 \%$ & \\
\hline \multirow{6}{*}{ Subcritério } & Compromisso da Direção & $18,49 \%$ & 3 & $10,78 \%$ & 3 \\
\hline & Cultura da Qualidade & $14,13 \%$ & 5 & $8,24 \%$ & 5 \\
\hline & MC e inovação & $18,27 \%$ & 4 & $10,65 \%$ & 4 \\
\hline & Sistema da Qualidade & $18,69 \%$ & 2 & $10,90 \%$ & 2 \\
\hline & Benchmarking & $6,51 \%$ & 6 & $3,79 \%$ & 10 \\
\hline & Planejamento Estratégico & $24,21 \%$ & 1 & $14,11 \%$ & 1 \\
\hline Critério & Fatores táticos & $22,84 \%$ & & $22,84 \%$ & \\
\hline \multirow{7}{*}{ Subcritério } & Incentivo aos Trabalhadores & $12,24 \%$ & 5 & $2,79 \%$ & 14 \\
\hline & $\begin{array}{l}\text { Envolvimento dos } \\
\text { Trabalhadores }\end{array}$ & $10,81 \%$ & 6 & $2,47 \%$ & 15 \\
\hline & Treinamento e Educação & $15,31 \%$ & 3 & $3,50 \%$ & 11 \\
\hline & Informações e Análise & $17,23 \%$ & 2 & $3,94 \%$ & 9 \\
\hline & Gestão de Fornecedores & $22,04 \%$ & 1 & $5,03 \%$ & 8 \\
\hline & Trabalho em Equipe & $8,28 \%$ & 7 & $1,89 \%$ & 16 \\
\hline & Comunicação & $14,07 \%$ & 4 & $3,21 \%$ & 12 \\
\hline Critério & Fatores operacionais & $18,86 \%$ & & $18,86 \%$ & \\
\hline \multirow{4}{*}{ Subcritério } & Design do Produto & $8,55 \%$ & 4 & $1,61 \%$ & 17 \\
\hline & Gestão de Processos & $31,98 \%$ & 2 & $6,03 \%$ & 7 \\
\hline & Foco no Cliente & $43,27 \%$ & 1 & $8,16 \%$ & 6 \\
\hline & $\begin{array}{c}\text { Gestão de Recursos } \\
\text { Humanos }\end{array}$ & $16,19 \%$ & 3 & $3,05 \%$ & 13 \\
\hline
\end{tabular}

Fonte: Autores

A partir dos resultados apresentados na Tabela 6, é possível observar a importância de cada atividade. Entre os três critérios, os fatores estratégicos 
apresentaram a maior prioridade (58,29\%), seguido pelos fatores táticos $(22,84 \%)$ e fatores operacionais (18,86\%). Tais resultados coincidiram com os apresentados por Talib, Rahman e Qureshi (2011), Souza et al. (2014) e Salgado et al. (2015).

Analisando essas evidências empíricas, verificou-se que os fatores determinantes na implantação da Gestão da Qualidade Total no estudo foram os de natureza estratégica. Por consequência, a máxima prioridade deve ser dada a esses fatores, devido ao seu valor agregado superior aos demais, o que indica também sua importância na indústria farmacêutica. Em relação ao peso local dos fatores estratégicos, a priorização das atividades deve ser dada ao Planejamento Estratégico $(24,21 \%)$, seguido em ordem de importância pelo Sistema da Qualidade $(18,69 \%)$ e Compromisso da direção (18,49\%).

Resultados semelhantes foram obtidos por Souza et al. (2014) em um estudo focado também no setor farmacêutico, em que os três fatores estratégicos citados também apresentaram as maiores importâncias. No entanto, nesse trabalho, o Sistema da Qualidade apresentou maior prioridade, seguido do Compromisso da Direção e Planejamento Estratégico.

Também assim como Salgado et al. (2015), que aplicou o AHP para priorizar práticas do TQM em pequenas e médias empresas no Brasil, a prática de Benchmarking também apresentou a menor importância, por ser, como relatado pelos autores, muitas vezes desconhecido e portanto, não utilizado pela maioria das empresas do país.

As priorizações divergiram, entretanto, em relação ao trabalho de Talib, Rahman e Qureshi (2011). Neste estudo com profissionais na área de serviço, o Planejamento Estratégico apareceu em último lugar na classificação. Compromisso da Direção foi prioritário, com MC e Inovação e Cultura da Qualidade nos respectivos segundo e terceiro lugares na classificação. Segundo os autores, a explicação para o menor grau de importância dado ao Planejamento Estratégico estaria no fato de os avaliadores considerarem o fator apenas em curto prazo, e não em longo prazo, minimizando sua importância.

Em relação aos fatores táticos locais, a atividade Gestão de fornecedores foi o fator que apresentou maior importância, com 22,04\%, com a Informação e Análise $(17,23 \%)$ em $2^{\circ}$ lugar e o Treinamento e Educação (15,31\%) em 3o lugar. Para Talib, Rahman e Qureshi (2011), a Gestão de Fornecedores, no entanto, apareceu 
na última colocação no ranking dos fatores táticos locais. A divergência em relação aos resultados do presente estudo pode ser explicada devido à diferença no setor estudado: na área de serviços, este critério não é tão significativo quando comparado a uma indústria transformadora, como a farmacêutica.

Finalmente, analisando os fatores operacionais, o Foco no cliente, com $43,27 \%$, apresentou a maior prioridade, seguido respectivamente pela Gestão de processos (31,98\%), e Gestão de recursos humanos (16,19\%). Tais resultados foram ao encontro do estudo realizado por Souza et al. (2014). A razão pela qual estes resultados convergiram pode estar no fato que os estudos seguiam o mesmo ramo (indústria farmacêutica), país e região estudada.

Com relação ao peso global dessas práticas do TQM, as avaliações de priorização ocorreram na seguinte ordem: o Planejamento Estratégico foi o fator mais significativo, com peso $14,11 \%$, e o Sistema da Qualidade e Compromisso da Direção apresentaram respectivamente, pesos de 10,90 \% e 10,78\%. Estas três práticas também foram as prioritárias para Souza et al. (2014), porém o Sistema da Qualidade apareceu na primeira colocação global, seguido por Compromisso da Direção e Planejamento Estratégico.

A presente classificação global das prioridades diferiu do trabalho de Kumar et al. (2011), em que Melhoria Contínua, Satisfação do Cliente e Trabalho em Equipe foram avaliados, respectivamente, como os mais importantes, no setor de manufatura. Visto que ambos os trabalhos foram realizados no mesmo ramo industrial, percebe-se a importância de se considerar também outras características como a diferença do número de subcritérios aplicados e o país estudado.

Analisando a Tabela 6, no ranking das priorizações das práticas de TQM, observa-se que as atividades avaliadas como menos significativas foram Design do produto, com peso de 1,61\%, e Trabalho em equipe, com 1,89\%. Esses fatores também obtiveram baixa classificação no estudo global realizado por Souza et al. (2014). Em contrapartida, Chen e Chen (2009), em um estudo uma indústria de base biotecnológica, afirmaram que o fator Trabalho em equipe apresentava alta prioridade.

Lewis (2005) realizou um estudo em pequenas e médias empresas certificadas pela norma ISO 9001 na América Central e notou que a Cultura da Qualidade foi um fator que apresentou baixo peso na classificação. Esse resultado 
diferiu do presente trabalho, em que a Cultura da Qualidade foi prioritária, alcançando a quinta colocação na classificação geral.

Para que este trabalho alcançasse resultados consistentes e satisfatórios foi considerado um limite superior do índice de consistência de 10\%. Tal valor corresponde à medida da consistência da matriz de comparações, especificado pelo método AHP.

\section{CONCLUSÕES}

As indústrias farmacêuticas desempenham um papel essencial no crescimento e qualidade de vida de uma população. Em uma área com grande importância e competitividade, elas estão sempre visando acompanhar e adequar-se as constantes inovações tecnológicas, buscando desenvolver novas tecnologias para aperfeiçoar seus processos produtivos e garantir vantagens no mercado. A qualidade nos produtos e processos na indústria farmacêutica é imprescindível e resultados satisfatórios requerem um conjunto de atitudes, comprometimento, comportamentos e conhecimentos de toda a organização.

As práticas de TQM representam um grande suporte às indústrias que buscam avaliar e melhorar seu desempenho, e com isso produzir medicamentos com confiáveis, com qualidade e acessíveis aos consumidores. A priorização das práticas do TQM auxilia empresas a avaliarem suas práticas atuais, buscando sempre uma gestão orientada a criar a consciência de qualidade em todos os processos organizacionais.

A ferramenta multicriterial AHP auxiliou na determinação dos elementos da Qualidade Total a serem priorizados no setor farmacêutico. Na avaliação dos profissionais da indústria farmacêutica entrevistados, os fatores estratégicos da TQM deveriam ser priorizados, com importância especial dentro desses fatores ao Planejamento Estratégico, Sistema da Qualidade e o Compromisso da Direção.

Cabe destacar, no entanto, as limitações desta pesquisa, relacionadas à impossibilidade de generalização. Os resultados apresentados neste estudo baseiam-se no julgamento de 12 especialistas de vários setores produtivos, no entanto apenas de uma indústria do setor farmacêutico. E como evidenciado, devido às diferenças nas classificações dos fatores de sucesso entre os setores, a 
aplicabilidade desse estudo se restringe, de forma mais específica, à indústria farmacêutica.

Como sugestão para trabalhos futuros estaria replicar essa pesquisa em mais empresas dentro no setor farmacêutico, visando obter conclusões mais amplas e sólidas. Além disso, poderia ser realizado o mesmo estudo utilizando outras ferramentas de tomada de decisão como, por exemplo, o TOPSIS. Outro estudo interessante seria aplicar a mesma estrutura hierárquica utilizando as ferramentas de forma combinada.

O modelo AHP desenvolvido neste trabalho permite uma melhor visualização das práticas de TQM e serve como um direcionamento para que outras indústrias farmacêuticas apliquem esse método, alcançando o máximo de benefícios para uma prática individual.

\section{REFERÊNCIAS}

ANAND, G.; KODALI, R. Selection of lean manufacturing systems using the PROMETHEE. Journal of Modelling in Management, v. 3, n. 1, p.40-70, 2008. http://dx.doi.org/10.1108/17465660810860372

BALLIS, A.; MAVROTAS, G. Freight village design using the multicriteria method PROMETHEE. Operational Research, v. 17, n. 2, p.213-232, 2007. http://dx.doi.org/10.1007/BF02942388

BASAK, I.; SAATY, T. L. Group decision making using the Analytic Hierarchy Process. Mathematical and Computer Modelling, v.17, n.4, p.101-109, 1993. http://dx.doi.org/10.1016/0895-7177(93)90179-3

BAUCHNER, H.; FONTANAROSA, P. B. Restoring Confidence in the Pharmaceutical Industry. The Journal of the American Medical Association, v. 309, n.6, p.607-609, 2013. http://dx.doi.org/doi:10.1001/jama.2013.58

BAYAZITA, O.; KARPAK, B. An analytical network process-based framework for successful total quality management (TQM): An assessment of Turkish manufacturing industry readiness. International Journal of Production Economics, v. 105, n. 1, p. 79-96, 2007. http://dx.doi.org/10.1016/j.ijpe.2005.12.009

BOTTANI, E.; E RIZZI, A. A fuzzy TOPSIS methodology to support outsourcing of logistics services. Supply Chain Management: An International Journal, v.11, n. 4, p.294-308, 2006. http://dx.doi.org/10.1108/13598540610671743

BRAGLIA, M.; CARMIGNANI, G.; FROSOLINI, M.; GRASSI, A. AHP-based evaluation of CMMS software. Journal of Manufacturing Technology Management, v. 15, n. 5, p.585 602, 2006. http://dx.doi.org/10.1108/17410380610668531

CAMPOS, V. F. Gerenciamento da rotina do dia a dia. INDG Tecnologia e Serviços LTDA. Belo Horizonte, 2004. 
CAMPOS, V. F. TQC: controle da qualidade total (no estilo japonês). Belo Horizonte: Fundação Christiano Ottoni, 1992.

CARVALHO, M. M.; PALADINI, E. P. Gestão da qualidade: teoria e casos. Rio de Janeiro: Elsevier, 2005.

CASTROGIOVANNI, G. et al. Where to acquire knowledge: Adapting knowledge management to financial institutions. Journal of Business Research, v. 69, p. 1812-1816, 2015. http://dx.doi.org/10.1016/i.jbusres.2015.10.061

CAVALCANTE, C.A.V.; ALMEIDA, A.T. Modelo multicritério de apoio a decisão para o planejamento de manutenção preventiva utilizando promethee II em situações de incerteza. Pesquisa Operacional, v. 25, n. 2, p.279-296, 2005. http://dx.doi.org/10.1590/S010174382005000200007

CHANG, W.L. Using multi-criteria decision aid to rank and select co-branding partners: From a brand personality perspective. Kybernetes, v. 38, n.6, p. 950-965, 2009.

http://dx.doi.org/10.1108/03684920910973171

CHEN, J.K., CHEN, I.S. TQM measurement model for the biotechnology industry in Taiwan. Expert Systems with Applications, v.36, n.5, p. 8789-8798, 2009.

http://dx.doi.org/10.1016/i.eswa.2008.11.013

CHIN, K.-S. et al. An AHP based study of critical factors for TQM implementation in Shanghai manufacturing industries. Technovation, v. 22, n. 11, p. 707-715, 2002. http://dx.doi.org/10.1016/S0166-4972(01)00065-7

COLLINS, T.R.; ROSSETTI, M.D.; NACHTMANN, H.L.; OLDHAM, J.R. The use of multiattribute utility theory to determine the overall best-in-class performer in a benchmarking study. Benchmarking: An International Journal, v.13, n.4, p.431- 446, 2006.

http://dx.doi.org/10.1108/14635770610676281

COSTA, J. F.S.; RODRIGUES, M.M.; MACHADO, A.P. Utilização do método de análise hierárquica (AHP) para escolha de interface telefônica. In: ENCONTRO NACIONAL DE ENGENHARIA DE PRODUÇÃO, 28, 2008, Rio de Janeiro. Anais...Rio de Janeiro, 2008.

EHIGIE, B.O.; MCANDREW, E.B. Innovation, diffusion and adoption of total quality management (TQM). Management Decision, v.43, n.6, p.925-940, 2005.

http://dx.doi.org/10.1108/00251740510603646

EISENHARDT, K. M. Building theories from case study research. The Academy of Management Review, v. 14, n. 4, p. 532-550, 1989.

http://dx.doi.org/10.5465/AMR.1989.4308385

FASSOULA, D. Transforming the supply chain. Journal of Manufacturing Technology Management, v.17, n.6, p.848-860, 2006. http://dx.doi.org/10.1108/17410380610678837

FOTOPOULOS, C. V.; PSOMAS, E. L. The structural relationships between TQM factors and organizational performance. The TQM Journal, v. 22, n. 5, p. 539-552, 2010. http://dx.doi.org/10.1108/17542731011072874

FRIEDLI, T.; GOETZFRIED, M.; BASU, P. Analysis of the implementation of total productive maintenance, total quality management, and just-in-time in pharmaceutical 
manufacturing. Journal of Pharmaceutical Innovation, v. 5, n. 4, p. 181-192, 2010. http://dx.doi.org/10.1007/s12247-010-9095-x

GOMES, L. F.; GOMES, C. F.; Almeida, A. T. Tomada de decisão gerencial: enfoque multicritério. Atlas, S. Paulo, 2009.

GUPTA, S. et al. Analytic hierarchy process (AHP) model for evaluating sustainable manufacturing practices in Indian electrical panel industries. Procedia-Social and

Behavioral Sciences, v. 189, p. 208-216, 2015.

http://dx.doi.org/10.1016/i.sbspro.2015.03.216

HARRINGTON, H.J., VOEHL, F., WIGGIN, H. Applying TQM to the construction industry. The TQM Journal, v. 24 n. 4, p. 352-362, 2012.

http://dx.doi.org/10.1108/17542731211247373

HSU, Y. G.; TZENG, H.; SHYU, J. Fuzzy multiple criteria selection of government sponsored frontier technology R\&D projects. R\&D Management, v. 33, n.5, p. 539-551, 2003.

http://dx.doi.org/10.1111/1467-9310.00315

HUNG, R. Y. Y. et al. Impact of TQM and organizational learning on innovation performance in the high-tech industry. International Business Review, v. 20, n. 2, p. 213-225, 2011. http://dx.doi.org/10.1016/.i.ibusrev.2010.07.001

JAMALI, R.; NEJATI,M. Women's career progression barriers and organizational justice: a study of Iranian society. Business Strategy Series, v.10, n.5, p.311-328, 2009.

http://dx.doi.org/10.1108/17515630910989204

KAHRAMAN, C.; ATES, N.Y.; CEVIK, S.; GULBAY, M.; ERDOGAN, S.A. Hierarchical fuzzy TOPSIS model for selection among logistics information technologies. Journal of

Enterprise Information Management, v.20, n.2, p.143-168, 2007.

http://dx.doi.org/10.1108/17410390710725742

KANG, H. Y.; LEE, H. I. Priority mix planning for semiconductor fabrication by fuzzy AHP ranking. Expert Systems with Applications, v. 32, n. 2, p. 560-570, 2007.

http://dx.doi.org/10.1016/j.eswa.2006.01.035

KHANNA, H. K.; SHARMA, D.D.; LAROIYA, S.C. Identifying and ranking critical success factors for implementation of Total Quality Management in the Indian manufacturing industry using TOPSIS. Asian Journal on Quality, v.12, n.1, p. 124-138, 2011.

http://dx.doi.org/10.1108/15982681111140598

KOLA, I.; LANDIS, J. Can the pharmaceutical industry reduce attrition rates?. Nature Reviews Drug Discovery, v. 3, n. 8, p. 711-716, 2004. http://dx.doi.org/10.1038/nrd1470

KOUTROUMANIDIS. T.; PAPATHANASIOU, J.; MANOS, B. A Multicriteria Analysis of Productivity of Agricultural Regions of Greece. Operational Research, v.2, n.3, p.339-346, 2002. http://dx.doi.org/10.1007/BF02936389

KUMAR, R., GARG, D., GARG, T.K. TQM success factors in North Indian manufacturing and service industries. The TQM Journal, v.23, n.1, p.36 - 46, 2011.

http://dx.doi.org/10.1108/17542731111097470 
LAU, A.W.T; TANG, S. L.; LI, Y. S. The level of TQM application by construction contractors in Hong Kong. International Journal of Quality \& Reliability Management, v. 32, n. 8, p. 830-862, 2015. http://dx.doi.org/10.1108/IJQRM-07-2013-0123

LEITE, I.M.S.; FREITAS, F.F.T. Análise comparativa dos métodos de apoio multicritério. A decisão: AHP, ELECTRE e PROMETHEE. In: XXXII ENCONTRO NACIONAL DE ENGENHARIA DE PRODUÇÃO. Anais... Rio Grande do Sul, 2012.

LEWIS, W.G., PUN, K.F., LALLA, T.R.M. An AHP-based study of TQM benefits in ISO 9001 certified SMEs in Trinidad and Tobago. The TQM Magazine, v. 17, n.6, p.558-572, 2005. http://dx.doi.org/10.1108/09544780510627651

LÓPEZ, J.C.L.; SÁNCHEZ, L.D.; CONTRERAS, M.A.A. A multicriteria decision support system with an evolutionary algorithm for deriving final ranking from a fuzzy outranking relation. Operations Research, v.8, n.1, p.47-62, 2008. http://dx.doi.org/10.1007/s12351$\underline{008-0006-6}$

MARTINS, R. A. Princípios da pesquisa científica. In: MIGUEL, P.A.C. (Org.). Metodologia de pesquisa em Engenharia de Produção e Gestão de Operações. 1.ed. Rio de Janeiro, Campus/Elsevier, p. 5-29, 2010.

MCADAM, R.; BARRON, N. The role of quality management in pharmaceutical development: clinical trials analysis. International Journal of Health Care Quality Assurance, v. 15, n. 3, p. 106-123, 2002. http://dx.doi.org/10.1108/09526860210426982

MAZUMDER, B.; BHATTACHARYA, S.; YADAV, A. Total quality management in pharmaceuticals: a review. International Journal of PharmTech Research, v.3, n.1, p.365375, 2011. http://www.sphinxsai.com/Vol.3No.1/pharm janmar11/pdf/JM11(PT=63)\%20pp\%20365-375.pdf

MEHRALIAN, G. et al. TOPSIS approach to prioritize critical success factors of TQM: evidence from the pharmaceutical industry. The TQM Journal, v. 28, n. 2, 2015. http://dx.doi.org/10.1108/TQM-03-2014-0028

MIGUEL, P. A. C. Estudo de caso na engenharia de produção: estruturação e recomendações para sua condução. Revista Produção, v. 17, n.1, p. 216-229, 2007. http://dx.doi.org/10.1590/S0103-65132007000100015

MIRANDA SILVA, G. et al. The role of TQM in strategic product innovation: an empirical assessment. International Journal of Operations \& Production Management, v. 34, n. 10, p. 1307-1337, 2014. http://dx.doi.org/10.1108/IJOPM-03-2012-0098

MOSADEGHRAD, A.M. Obstacles to TQM success in health care systems. International Journal of Health Care Quality Assurance, v. 26, n. 2, p. 147-173, 2013.

http://dx.doi.org/10.1108/09526861311297352

MOTA, C.M.M.; ALMEIDA, A.T. Método multicritério electre IV-H para priorização de atividades em projetos. Pesquisa Operacional, v.27, n.2, p.247 - 269, 2007. http://dx.doi.org/10.1590/S0101-74382007000200004

NADAE, J.; OLIVEIRA, J.A.; OLIVEIRA, O.J.; Um estudo sobre a adoção dos programas e ferramentas da qualidade em empresas com certificação ISO 9001: estudo de casos múltiplos. GEPROS, v.4, n.4, p.93-114, 2009.

http://revista.feb.unesp.br/index.php/gepros/article/download/852/283 
NEJATI, M.; NEJATI, M. Ranking airlines' service quality factors using a fuzzy approach: study of the Iranian society. International Journal of Quality \& Reliability Management, v.26, n.3, p. 247-260, 2009. http://dx.doi.org/10.1108/02656710910936726

NIKOU, S.; MEZEI, J. Evaluation of mobile services and substantial adoption factors with Analytic Hierarchy Process (AHP). Telecommunications Policy, v. 37, n.10, p.915-929, 2013. http://dx.doi.org/10.1016/j.telpol.2012.09.007

OOI, K. et al. Are TQM practices supporting customer satisfaction and service quality?. Journal of Services Marketing, v. 25, n. 6, p. 410-419, 2011. http://dx.doi.org/10.1108/08876041111161005

POLYCHRONIOU, P.V.; GIANNIKOS, I. A fuzzy multicriteria decision-making methodology for selection of human resources in a Greek private bank. Career Development International, v.14, n.4, p.372-387, 2009.http://dx.doi.org/10.1108/13620430910979853

RANGEL, L.A.D., GOMES, L.F.A.M., MOREIRA, R.A. Decision theory with multiple criteria: An application of ELECTRE IV and TODIM to SEBRAE/RJ. Pesquisa Operacional, v. 29, p. 577- 590, 2009. http://dx.doi.org/10.1590/S0101-74382009000300007

SAATY, T. L. The analytic hierarchy process and health care problems. New York: McGraw-Hill.1980.

SAATY, T. L.; OZDEMIR, M. S. The Encyclicon: a dictionary of decisions with dependence and feedback based on the Analytic Network Process. Pittsburgh: RWS, 2005.

SAATY, T. L.;VARGAS L. G: Prediction, Projection and Forecasting in Applications of the Analytical Hierarchy Process in Economics, Finance, Politics, Games and Sports (Kluwer Academic Publishers, Boston), Universidad the California, Kluwer Academic Publishers, 1991.

SAATY, T.L. The analytic hierarchy process: planning, priority setting, resource allocation. New York, NY: McGraw-Hill, 1980.

SAATY, T.L. The analytic hierarchy process. New York, NY: Pergamon Press, 1988.

SALGADO, E.G.; SALOMON, V.A.P.; MELLO, C.H.P.Analytic hierarchy prioritization of new product development activities for electronics manufacturing. International Journal of Production Research, v.50, n.17, p.4860-4866, 2012.

http://dx.doi.org/10.1080/00207543.2012.657972

SALGADO, E. G. Modelo de referência para o processo de desenvolvimento de produtos eletrônicos em empresas de base tecnológica: estudos de casos múltiplos com decisão multicriterial. Tese (Doutorado)- Universidade Estadual Paulista, Faculdade de Engenharia de Guaratinguetá, 2011.

SALGADO, E. G. et al. An analytic hierarchy process analysis for small and medium sized enterprises: Prioritizing the practices of Total Quality Management in Brazil. International Journal for Quality Research, v. 9, n. 2, p.185-196, 2015. http://www.ijgr.net/journal/v9$\underline{\text { n2/1.pdf }}$

SALMERON, J. L.; HERRERO, I. An AHP-based methodology to rank critical success factors of executive information systems. Computer Standards \& Interfaces, v. 28, n. 1, p. 1-12, 2005. http://dx.doi.org/10.1016/..csi.2004.09.002 
SARATHY, P. SANJAY. TQM practice in real-estate industry using AHP. Quality \& Quantity, v.47, p. 2049-2063. 2013. http://dx.doi.org/10.1007/s11135-011-9641-8

SCHNEIDER, U. et al. Operational Excellence in Practice-the Application of a Takt-Time Analysis in Pharmaceutical Manufacturing. Journal of Pharmaceutical Innovation, v. 10, n. 2, p. 99-108, 2015. http://dx.doi.org/10.1007/s12247-014-9210-5

SHIBA, S.; GRAHAM, A.; WALDEN, D.TQM: Quatro Revoluções na Gestão da Qualidade, Ed. Bookman. 1997.

SHIMIZU, T. Decisão nas organizações. São Paulo. Editora Atlas, 2006.

SINGH, S.B.; DHALLA, R. S. Effect of total quality management on performance of indian pharmaceutical industries. International Conference on Industrial Engineering and Operations Management. Bangladesh, 2010.

SINGH, R.P.; NACHTNEBEL, H. P. Analytical hierarchy process (AHP) application for reinforcement of hydropower strategy in Nepal. Renewable and Sustainable Energy Reviews, v. 55, p. 43-58, 2016. http://dx.doi.org/10.1016/j.rser.2015.10.138

SOUZA Jr., C.A.; SALGADO, E. G.; NEVES, F.O.; ALVARENGA, A. D. Priorização das práticas de TQM na indústria farmacêutica. Espacios, v. 35, n.4, p.7, 2014. http://www.revistaespacios.com/a14v35n04/14350407.html

TALIB, F., RAHMAN, Z., QURESHI, M.N. Prioritizing the practices of total quality management: An analytic hierarchy process analysis for the service industries. Total Quality Management \& Business Excellence, v.22, n.12, p.1331-1351, 2011. http://dx.doi.org/10.1080/14783363.2011.625192

TAM, C.M.; TONG, T.K.L.; LAU, C.T. ELECTRE III in evaluating performance of construction plants: case study on concrete vibrators. Construction Innovation, v.3, p.45-61, 2003. http://dx.doi.org/10.1108/14714170310814846

TRAMARICO, L.C.; SALOMON, P. A. V.; MARINS S. A. F. Modelagem com AHP e BOCR para Seleção de Prestadores de Serviços Logísticos. Revista eletrônica Pesquisa Operacional para Desenvolvimento, v.4, n.2, p.139-159, 2012.

http://www.podesenvolvimento.org.br/inicio/index.php?journal=podesenvolvimento\&page=art icle\&op=view\&path\%5B\%5D=99\&path\%5B\%5D=166

VOSS, C.; TSIKRIKTSIS, N.; FROHLICH, M. Case research in operations management. International Journal of Operations \& Production Management, v. 22, n. 2, p. 195-219, 2002. http://dx.doi.org/10.1108/01443570210414329

YIN, R. Estudo de caso. Planejamento e métodos. 3. ed. Porto Alegre: Bookman, 2005.

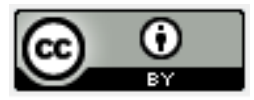

Artigo recebido em 31/01/2015 e aceito para publicação em 27/04/2016 DOI: http://dx.doi.org/ 10.14488/1676-1901.v16i2.1978 